\title{
Tricuspid Valve Dysplasia in a Dog
}

\author{
Gabriela de Carvalho Cid', Luciano da Silva Alonso', Ana Paula de Castro Pires', Mariana Sequeira d'Avila', \\ Taïna Gonçalves ${ }^{3}$, Nathália da Silva Carvalho', Vivian de Assunção Nogueira' \& Ticiana Nascimento França'
}

\begin{abstract}
Background: Congenital cardiac diseases are a common cause of death in puppies. Tricuspid valve dysplasia is characterized by thickening and displacement of the leaflets of the tricuspid valve, agenesis of the valves, and incomplete separation of valve components. Papillary muscles may fuse and display shortened or absent chordae tendineae that contribute to tricuspid regurgitation. Diagnostic features of tricuspid valve dysplasia include cardiomegaly with massive right atrium enlargement on thoracic radiography and tricuspid insufficiency on an ultrasound. We aimed to describe clinicopathological findings in a dog (Canis familiaris) with tricuspid dysplasia.

Case: We aimed to describe tricuspid valve dysplasia in a dog referred for necropsy at the Anatomical Pathology Sector of The Rural Federal University of Rio de Janeiro, Brazil, with a clinical history of abdominal swelling, dyspnea, cyanosis, ascites, and prostration. Echocardiography and abdominal ultrasound revealed right ventricular enlargement, hepatomegaly, and splenomegaly. Examination of the heart showed prominent enlargement, thickening and dilation of the right chambers, thickening of the tricuspid leaflets, and moderately shortened chordae tendineae. The liver was enlarged, with a nutmeg pattern, and foci of clotting and fibrin adhesions in the lateral right lobule.

Discussion: Epidemiological, clinical, and pathological findings were consistent with tricuspid valve dysplasia. Although structural abnormalities of the tricuspid and mitral valves are well known in fetuses and neonates, congenital and secondary tricuspid malformations are rare in dogs. The survival rate is associated with the severity of heart lesions. Tricuspid valve dysplasia is mostly observed in large-breed dogs ( $>20 \mathrm{~kg})$, particularly in Labrador Retrievers, Boxers, and German Shepherds. Regardless, most dogs with tricuspid valve dysplasia are of a pure-breed, which differs from our findings because our dog was a mongrel. Our dog displayed signs of dyspnea, cyanosis, abdominal swelling, prostration, and enlarged liver and spleen on ultrasound examination. Tricuspid valve dysplasia led to heart enlargement and right congestive heart failure, with consequent ascites, abdominal swelling, weakness, lethargy, jugular venous distension, and hepatomegaly. Overall, the heart showed prominent enlargement, thickening and dilation of the right chambers, thickening of the tricuspid leaflets, and moderately shortened chordae tendineae. The liver had a nutmeg pattern. Tricuspid valve dysplasia is characterized by malformation of the tricuspid valve leaflets, chord tendineae, or papillary muscles. Malformed tricuspid valves are known to result in variable degrees of regurgitation, leading to right atrial overflow and ventricular eccentric hypertrophy. Differential diagnosis includes myocarditis, tricuspid valve endocarditis, tricuspid endocardiosis, tricuspid valve prolapse and right ventricular dysplasia, right ventricular enlargement with tricuspidal regurgitation due to pulmonary insufficiency, and arrhythmogenic right ventricular cardiomyopathy. Signs of heart murmurs (irregular sounds of the heart) on clinical examination may indicate an irregular blood flow pattern, and imaging tests may be necessary for assessing the presence and severity of any lesions. The epidemiologic, clinical, and pathological findings were consistent with those of tricuspid valve dysplasia.
\end{abstract}

Keywords: dysplasia, tricuspid, malformation. 


\section{INTRODUCTION}

Congenital cardiac diseases are a common cause of death in puppies. Tricuspid valve dysplasia is characterized by thickening and displacement of the leaflets of the tricuspid valve, agenesis of the valves, and incomplete separation of valve components. Papillary muscles may fuse and display shortened or absent chordae tendineae that contribute to tricuspid regurgitation. Tricuspid valve dysplasia is rare, particularly when compared with other cardiac congenic malformations, such as the tetralogy of Fallot, pulmonary and aortic stenosis, and patent ductus arteriosus [2,11].

According to Oliveira et al. [7] tricuspid valve dysplasia is most common in young, male, large purebred dogs, such as Labrador Retriever, Boxer, and German Shepherds. Clinical signs include exercise intolerance, pale mucosa, ascites, and higher systolic murmur at the right apex. Diagnostic features of tricuspid valve dysplasia include cardiomegaly with massive right atrium enlargement on thoracic radiography and tricuspid insufficiency on an ultrasound [10].

Medical therapy is palliative and aims to improve the quality of life. Standard procedures include periodic thoracentesis and abdominocentesis. The only definitive cure for tricuspid valve dysplasia is valve replacement surgery, which has been successfully performed in only few dogs $[3,9]$. We aimed to describe the clinicopathological findings in a dog with tricuspid dysplasia.

\section{CASE}

A 5-month-old, mongrel domestic dog was admitted to the emergency service of the Small Animals Veterinary Hospital (HVPA) of the Rural Federal University at Rio de Janeiro (UFRRJ); it presented with a swollen abdomen. Echocardiography and abdominal ultrasound examination showed right ventricular enlargement, hepatomegaly, and splenomegaly. The dog died during the procedures because of a cardiorespiratory arrest and then, it was referred for a necropsy.

Gross examination revealed a low body score (1.5/5), oral and ocular pale mucosa, remarkable abdominal swelling, thoracic and abdominal subcutaneous edema, subcutaneous hemorrhage, splenomegaly, and mildly reddish and clear liquid in the thorax. Axillary lymph nodes were mildly swollen. Lungs were rubbery and dark pink in color with an extensive area of emphysema and foci of atelectasis. Foamy reddish liquid was found in the trachea. The liver was enlarged, with a nutmeg pattern and foci of clotting and fibrin adhesions in the lateral right lobule. Heart examination showed prominent enlargement (Figure 1A), thickening and dilation of the right chambers, thickening of the tricuspid leaflets, and moderately shortened chordae tendineae (Figure 1B, 1C and 1D).

\section{DISCUSSION}

Epidemiological, clinical, and pathological findings were consistent with tricuspid valve dysplasia. Although structural abnormalities of the tricuspid and mitral valves are well known in fetuses and neonates, congenital and secondary tricuspid malformations are rare in dogs [6]. Tricuspid valve dysplasia is mostly observed in large-breed dogs ( $>20 \mathrm{~kg}$ ), particularly in Labrador Retrievers, Boxers and German Shepherds. Because of a genetic predisposition, Labrador Retrievers are up to 35 times more prone to develop this condition than other breeds [7]. Regardless, most dogs with tricuspid valve dysplasia are of a pure-breed, which differs from our findings because our dog was a mongrel $[4,7]$.

The survival rate is associated with the severity of heart lesions. Some dogs may survive many years with tricuspid valve dysplasia without displaying any clinical signs; however, once congestive heart failure is prominent, prognosis is poor. Our dog died at the age of 5 months, similar to the findings of other studies in which the survival rate ranged from 3-5 months $[5,10]$.

Most surveys point to a gender disposition in developing tricuspid valve dysplasia. Male dogs seem to be affected three times more than female dogs. However, little is known about this alleged breed disposition because most research lacks scientific grounds to justify those findings $[5,9]$.

Our dog displayed signs of dyspnea, cyanosis, abdominal swelling, prostration, and enlarged liver and spleen on ultrasound examination. Tricuspid valve dysplasia lead to heart enlargement and right congestive heart failure, with consequent ascites, abdominal swelling, weakness, lethargy, jugular venous distension, and hepatomegaly [9].

Overall, the heart showed prominent enlargement, thickening and dilation of the right chambers, thickening of the tricuspid leaflets, and moderately shortened chordae tendineae. The liver had a nutmeg pattern. Tricuspid valve dysplasia is characterized by 


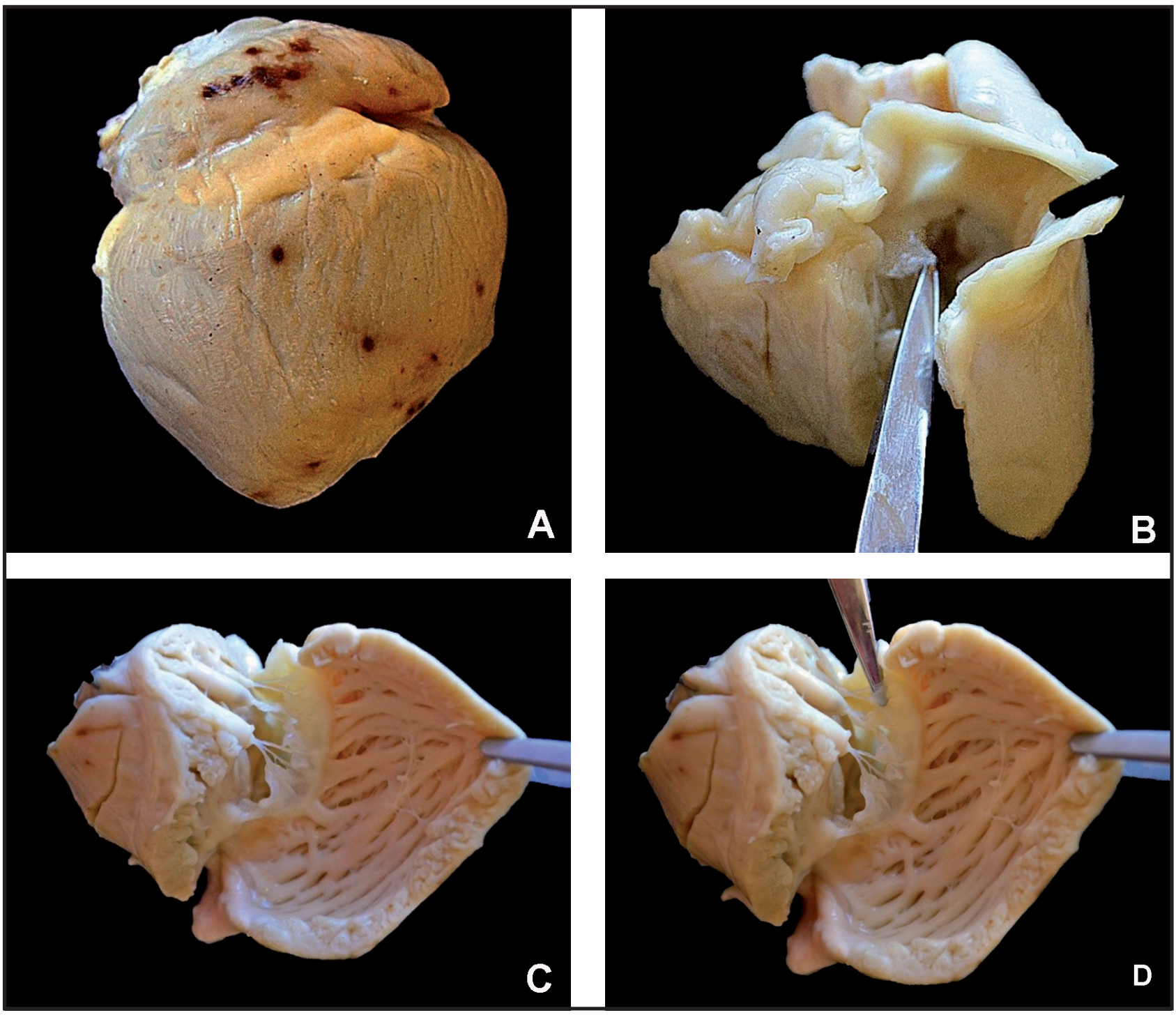

Figure 1. Valve Tricuspid Dysplasia in a Dog. A- Rounded heart, right ventricular enlargement, 4.4-cm-long right auricle, and foci of ecchymosis on the right side of the heart. B- Tricuspid valve. Parietal cusp shortened, thickened,, and opaque. C- Heart. Triscupid valve showing shortened, thickened, and opaque septal cusp. Moderately shortened cord tendine and papillary muscle hypertrophy. D- Heart. Triscupid valve shortened. Details of Figure C.

malformation of the tricuspid valve leaflets, chord tendineae, or papillary muscles. Malformed tricuspid valves are known to result in variable degrees of regurgitation, leading to right atrial overflow and ventricular eccentric hypertrophy [9].

Sousa et al. [10] reported two cases of tricuspid valve dysplasia in dogs and described ascites (reddish liquid in the abdomen), right ventricular eccentric hypertrophy, vena cava distension, shortened chordae tendineae, and abnormally thick tricuspid valve leaflets as the main morphological findings. These findings are similar to those observed in our case and in dogs in previous studies $[6,5]$.

During necropsy, we fixed the whole heart in $10 \%$ buffered formalin for better assessment of the gross changes. We established the diagnosis based on the heart morphological findings, and thus, histopathological examination was unnecessary. In addition, Park et al. [8] only described non-specific heart microscopically changes in a case of tricuspid valve dysplasia in a dog, such as disarray and disorganization of myocytes with yellow-brow pigments (lipofuscin).

Differential diagnosis includes myocarditis, tricuspid valve endocarditis, tricuspid endocardiosis, tricuspid valve prolapse and right ventricular dysplasia, right ventricular enlargement with tricuspidal regurgitation due to pulmonary insufficiency, and arrhythmogenic right ventricular cardiomyopathy. Signs of heart murmurs (irregular sounds of the heart) on clinical examination may indicate an irregular blood flow pattern, and imaging tests may be necessary for assessing the presence and severity of any lesions. 
Electrocardiography detects abnormal rhythm patterns in the heart, thoracic X-rays evaluate the heart shape and pulmonary edema, and ultrasound confirms ascites. However, the best diagnostic tool for tricuspid valve dysplasia is echocardiography [1]

Echocardiography is the gold standard for the diagnosis of tricuspid valve dysplasia. An echocardiographic examination allows assessment of the severity of lesions. Moreover, classifications are based on the degree of tricuspid leaflet deformation, regurgitation, and the size of the right ventricle [9]. In our study, echocardiography revealed right ventricular enlargement, making it essential to evaluate the best diagnostic management.

Tricuspid valve dysplasia treatment is aimed at improving the quality of life. Diuretic drugs can reduce abdominal swelling; however, most cases require long-term abdominocentesis. Anti-arrhythmics drugs can be used; however, majority dogs fail to develop rhythm abnormalities. Low sodium and cardiac diets are also used for treatment [9]. In our study, the dog died during the diagnostic procedures; therefore, the clinic did not have time to employ any medical treatment. Based on the signs of congestive heart failure, palliative treatment would have been the best option.
Valve replacement surgery is the only treatment that allows complete recovery from tricuspid valve dysplasia. Although largely performed in human medicine, this procedure is rarely performed in domestic species because of high costs and need for an extracorporeal circulation [9].

Tricuspid valve dysplasia is a rare condition in dogs, particularly when compared with other congenital cardiac diseases; however, it cannot be neglected and should always be considered during differential diagnosis of heart diseases in dogs.

Because of non-specific clinical signs associated with congestive heart failure, a meticulous physical examination and heart auscultation are mandatory. Heart murmurs, a potentially clear indication of abnormalities in the blood flow, are useful for a presumptive diagnosis of tricuspid valve dysplasia.

Ancillary exams are essential for a definitive diagnose, in particular, echocardiography, which is a gold standard test for tricuspid valve dysplasia. Modern diagnostic tools allow early diagnosis that permits effective treatments to be initiated immediately, increasing survival rates and the quality of life of affected dogs.

\section{REFERENCES}

1 Ammash N.M., Warnes C.A. \& Conolly H.M. 1997. Mimics of Ebstein's anomaly. American Heart Journal. 134: 508-513.

2 Becker A.E., Becker M.J. \& Edwards J.E. 1971. Pathologic spectrum of dysplasia of the tricuspid valve. Archives of Pathology. 91: 167-178.

3 Ettinger S.J. \& Feldman E.C. 2010. Tratado de Medicina Interna Veterinária. 7.ed. Rio de Janeiro: Guanabara Koogan, $2217 \mathrm{p}$.

4 Gregori T., Ochoa P.G. \& Quintavalla F. 2008. Congenital heart defects in dogs: a double retrospective study on cases from University of Parma and University of Zaragoza. Annali della Facoltà di Medicina Veterinaria. Università di Parma. 28: 79-90.

5 Larsson M.H.M.A., Pereira L.\& Kogika M.M. 1996. Displasia de tricúspide associada à anomalia de Ebstein em cão: relato de caso. Brazilian Journal of Veterinary Research and Animal Science. 33: 302-303.

6 Liu S.K. \& Tilley L.P. 1976. Dysplasia of the tricuspid valve in the dog and cat. Journal of the American Veterinary Medical Association. 169: 623-630.

7 Oliveira P., Domenech O., Silva J., Vannini S., Bussadori R. \& Bussadori C. 2011. Retrospective review of congenital heart disease in 976 dogs. Journal of Veterinary Internal Medicine. 25: 477-483.

8 Park C., Choi C.B. \& Sur J.H. 2004. Tricuspid valve dysplasia (TVD) in an American cocker spaniel dog. Korean Journal of Veterinary Research. 44: 125-130. 
9 Paslawska U., Noszczyk-nowak A., Janiszewski A. \& Nicpón J. 2013. Tricuspid dysplasia in dogs. Bulletin of the Veterinary Institute in Pulawy. 57: 123-126.

10 Sousa M.G., Gerardi D.G., Alves R.O. \& Camacho A.A. 2006. Tricuspid valve dysplasia and Ebstein's anomaly in dogs: case report. Arquivo Brasileiro de Medicina Veterinária e Zootecnia. 58: 762-767.

11 Tylley L.P. \& Goodwin J.K. 2002. Manual de Cardiologia para Cães e Gatos. 3.ed. São Paulo: Roca, 504p. 